\title{
Simulation and Experimental Verification of Ground Target Recognition Based on HRRP
}

\author{
Yingrui FAN ${ }^{\mathrm{a}}$, Zhongxiang LIU $^{\mathrm{b}}$, Zhiyong SONG, Qiang FU \\ ATR Key Laboratory, School of Electronic Science and Engineering, National University of Defense \\ Technology, Changsha 410073, China \\ aemial:570324520@qq.com, bemial 373161732@qq.com
}

Keywords: HRRP, Adaptive Segmentation, Ground Target Recognition, Experimental Verification

\begin{abstract}
Study on the ground target recognition for radar on multiple platforms based on SAR has been perfected gradually, however in view of the radar on missile platform, its velocity is high, and its trajectory is limited, using HRRP has advantages of strong real-time, low computational complexity compared to SAR. But, domestic ground target recognition for radar on missile platform based on HRRP still rest on the theory research stage. This paper uses GRECO to study HRRP inter-class separability, then uses an adaptive segmentation algorithm to deal with measured data outfield, avoiding the partly clutter's interference. Finally experiment results prove the feasibility of ground target recognition based on HRRP, has important significance for promoting the practical HRRP for ground target recognition.
\end{abstract}

\section{Introduction}

Ground target recognition in the ground attack, resource exploration, ground patrol, ground search and rescue, etc. has been widely used. The current ground target recognition has spaceborne, airborne, UAV-borne, missile-borne and other platforms[5]. Ground target recognition for spaceborne radar usually uses SAR images to detect resources, UAV usually carries optical rarar or SAR radar to accomplish ground target search and attack assessment[6], however missile-borne radar because of its limited trajectory usually uses HRRP's features to recognize target. With the improvement of the radar resolution, the target is no longer a point on the radial dimension, but extended to multiple point as a one-dimensional distance profile. HRRP can not only reflect the target's geometric shape and structure features, but also has characteristics of strong real-time and facile. It can be obtained from a cycle of echo. So it has a high value of practical application.

Though, domestic target recognition based on HRRP mostly aims at sea-surface target or aerial target[2,3]. On the one hand because there is no ground background measured data to research, on the other hand the sea-surface and air have a relatively clean background, compared to complex ground. They are easier to research. Pointing to ground target, Xie et al. in Xidian University researched missile-borne radar ground moving target classification based on micro-doppler feature, but didn't complish target recognition.

This paper starts from GRECO simulation data, adopts MCC-TMM, the simulation results show that single target's full HRRP has a good matching and distinction. In the fourth section, this article uses the adaptive segmentation for the field measured data, verifies the feasibility whether HRRP can be used for ground target recognition or not.

\section{Problem Description}

Compared with the single background of the sea-surface target and the aerial target, ground target's background has obvious unpredictability and variability. Grass, sand, cropland and the forest, etc. have completely different scattering characteristics. In addition, the swing of the leaves also increases the complexity of the background. So whether HRRP can be effectively used for ground target recognition is always controversial.

As shown in Figure 1, it is a typical recognition scene for ground target. $\mathrm{R}$ is the distance form 
the radar beam center to the ground, azimuth angle and elevation angle of the beam center respectively is $\theta, \boldsymbol{\alpha}$. Due to the randomness of the attack Angle and the diversity of the ground target formation, the radar beam area may contains multiple targets. The HRRP segmentation is the prerequisites of matching recognition. The segmentation method will be shown in section 4.1.

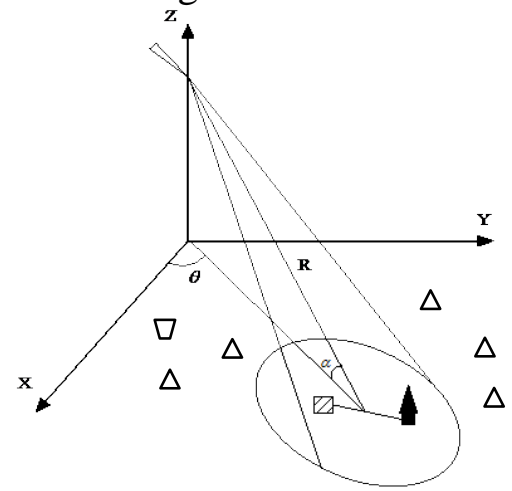

Fig.1. Ground target recognition scene

After segmentation, we extract a single target's HRRP, then recognize target type by means of MCC-TMM[1].

$$
\begin{aligned}
& p_{\bar{i}}=\max \left(x \operatorname{corr}\left(\frac{x}{\|x\|_{2}}, \frac{T_{\bar{i}}}{\left\|T_{i}\right\|_{2}}\right)\right) \quad i \in\left(0^{0}, 360^{\circ}\right) \\
& C=\overline{C_{y z}}+a \sigma_{y z}
\end{aligned}
$$

In equation (1), radar vehicle template library, T, one HRRP of $\mathrm{T}$ in azimuth $\mathrm{i}$, Ti, a test target's HRRP, $x$, the maximum of sliding correlation coefficient for $x$ and Ti, pi. Equation (2) describes the threshold, C, matched-mean-value in two different kinds of target, ${ }^{{ }^{y z}}$, matched var, ${ }^{\sigma z}$, factor seted by recognition rate, a. For the full-azimuth template library, choose the max pi to compare with the threshold $\mathrm{C}$, determine that whether the target $\mathrm{x}$ is a radar vehicle.

\section{Research on Inter-class Separability by GRECO}

In the ways of calculating electromagnetic scattering features for complex targets, graphic electromagnetic computing (GRECO) is one of the most effective methods to compute the RCS of complex targets. This way is widely used in theoretical analysis because of its strong real-time, small storage capacity and fast calculation.

As shown in Figure 2, this paper gets 3D model of crawler-type carrier vehicle, corner reflector and wheel-type phased array radar vehicle through 3D MAX, then computes three targets' HRRP in each azimuth under $30^{\circ}$ elevation angle, at last establishes three targets' full-azimuth template library in $30^{\circ}$ elevation angle.

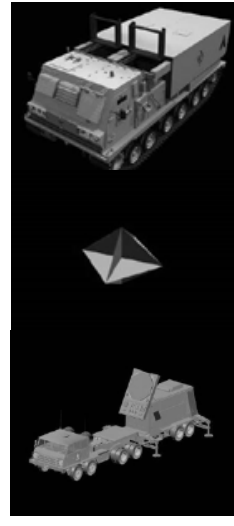

(a)

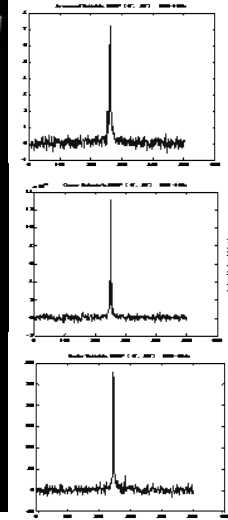

(b)

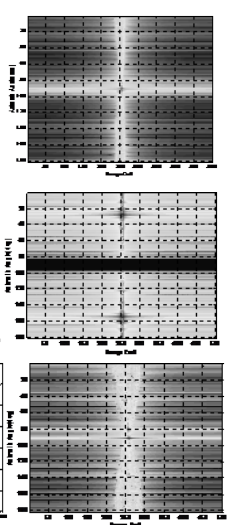

(c)

Fig.2. 3D model by 3D MAX and HRRP by GRECO, (a)3D model, (b) one HRRP(azi $=0^{\circ}$, ele $=30^{\circ}$, $\mathrm{SNR}=10 \mathrm{~dB}$ ), (c)full-azimuth template library, from top to bottom, carrier vehicle, corner reflector, radar vehicle 
Do an experiment, we choose randomly one HRRP in any azimuth under $30^{\circ}$ elevation, match it with radar vehicle template library, compare the results with the threshold, then conclude that whether the HRRP is radar vehicle's. So the problem comes, setting the threshold need ensure a good self-matching and inter-class separability. And furthermore, research on target HRRP's inter-class separability under clutter is instructive to set threshold in next measurement verification stage. Do the experiment in Monte carlo method, we get matched value's mean and var in any azimuth for three targets. Mean value is shown in Figure 3.

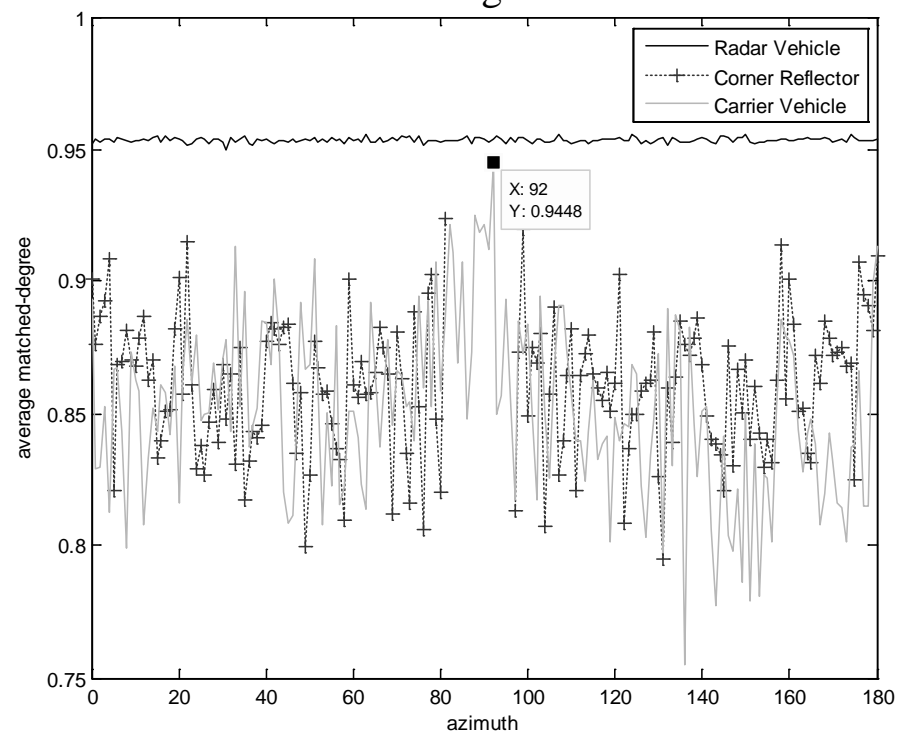

Fig.3. HRRP in full-azimuth matched-mean-value with radar vehicle template library(ele $=30^{\circ}$, $\mathrm{SNR}=10 \mathrm{~dB}$ ), from top to bottom, blue line is radar vehicle's, red dotted is corner reflector's, green line is carrier vehicle's

As shown in Figure 3, blue line is radar vehicle's full-azimuth matched result, mean is about 0.96, var is low, it has good self-matching. And the other targets' mean value is all under 0.95, most of them is under 0.9. So it has good inter-class separability. What is interesting is that their var is higher than radar vehicle's. Figure 3 inspires us that a new method for ground target recognition based on HRRP. When radar beam irradiate a target, we can get HRRP sequences for the same target. Finally, we judge target type by target HRRP sequences' var. Matched successfully, its var is low, otherwise it is high.

Table 1 lists radar vehicle's and carrier vehicle's matched value's mean and var in different SNR conditions. When SNR is $5 \mathrm{~dB}$, carrier vehicle's sequences var is 133 times higher than radar vehicle's. When SNR is $10 \mathrm{~dB}$, carrier vehicle's sequences var is 1290 times higher than radar vehicle's. It is positively related to SNR.

Table 1. Full-azimuth HRRP sequences matched value's mean and var

\begin{tabular}{c|c|c}
\hline \multirow{2}{*}{} & \multicolumn{2}{|c}{ SNR $=10 \mathrm{~dB}$} \\
\cline { 2 - 3 } & Radre Vehicle & Carrier Vehicle \\
\hline Mean & 0.9535 & 0.8521 \\
\hline Var & $8.5182 \mathrm{e}-007$ & 0.0011 \\
\hline & \multicolumn{2}{|c}{ SNR=5dB } \\
\hline Mean & Radre Vehicle & Carrier Vehicle \\
\hline Var & 0.8717 & 0.7802 \\
\hline
\end{tabular}




\section{Experimental Verification}

For real data in experimental verification, we did the high tower static experiment, got Isuzu truck's and Highlander SUV's echo data in multiple azimuth and elevation angle for ground background. The experiment scene is shown as Figure $4,{ }^{\theta}$ expresses azimuth angle and ${ }^{\alpha}$ expresses elevation angle. The experiment parameter is listed in Table 2. The test target and experiment scene's landform background are shown in Figure 5:

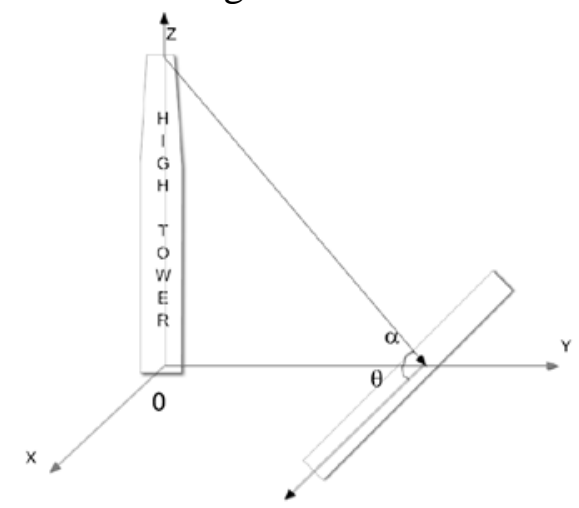

Fig. 4. Experiment scene

Table 2. Experiment parameter

\begin{tabular}{c|c}
\hline Intermediate frequency work mode & Stepped frequency pulse \\
\hline Operation bandwidth & $512 \mathrm{MHz}$ \\
\hline Stepped frequency interval & $0.5 \mathrm{MHz}$ \\
\hline Range resolution & $2930 \mathrm{~mm}$ \\
\hline Target 1 & Truck: $6745 * 1880 * 2220 \mathrm{~mm}$ \\
\hline Target 2 & SUV: $4795 * 1910 * 1730 \mathrm{~mm}$ \\
\hline Landform background & Construction, road, cropland, sand, grass, etc. \\
\hline Environment temperature & $0-50^{\circ} \mathrm{C}$ \\
\hline
\end{tabular}

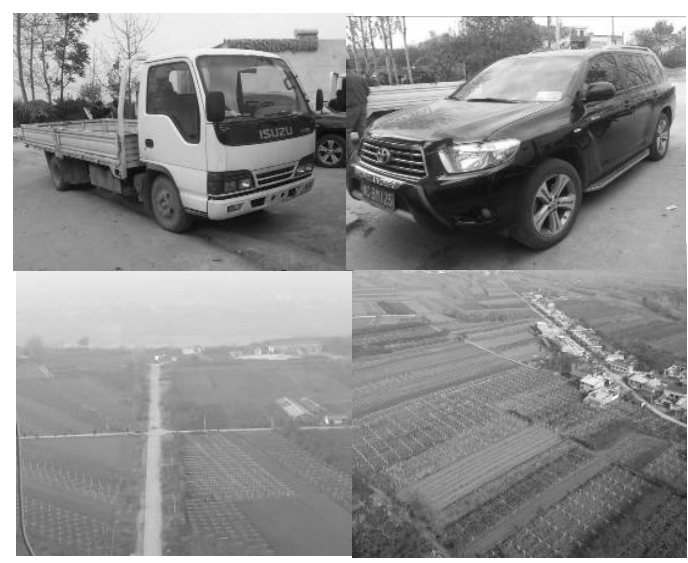

Fig.5. Test target and experiment scene's landform background, (a)truck, (b)SUV, (c-d)experiment scene's landform background

In experimentation, we choose the following elevation angle: $10^{\circ}, 15^{\circ}, 20^{\circ}, 25^{\circ}$ and $30^{\circ}$, the azimuth angle: $0^{\circ}, 45^{\circ}, 90^{\circ}, 135^{\circ}, 180^{\circ}, 225^{\circ}, 270^{\circ}$ and $315^{\circ}$, the polarization modes: horizontal polarization and vertical polarization. What's the most important, the background is time-varying.

\subsection{HRRP Adaptive Segmentation}

In the high tower static experiment, radar acquires R-D image. Then we filtering the image, ifft in azimuth dimension, in the end extract HRRP according to the peak position of the R-D image, 
just as shown in Figure 6-b. The distance between the two peaks is beyond the actual length of a truck. So the peak in 600 point can't express the architectural feature of the truck, without contribution to recognition. It's the clutter. Therefore we need HRRP segmentation to improve the recognition rate by avoiding the partly clutter interference.

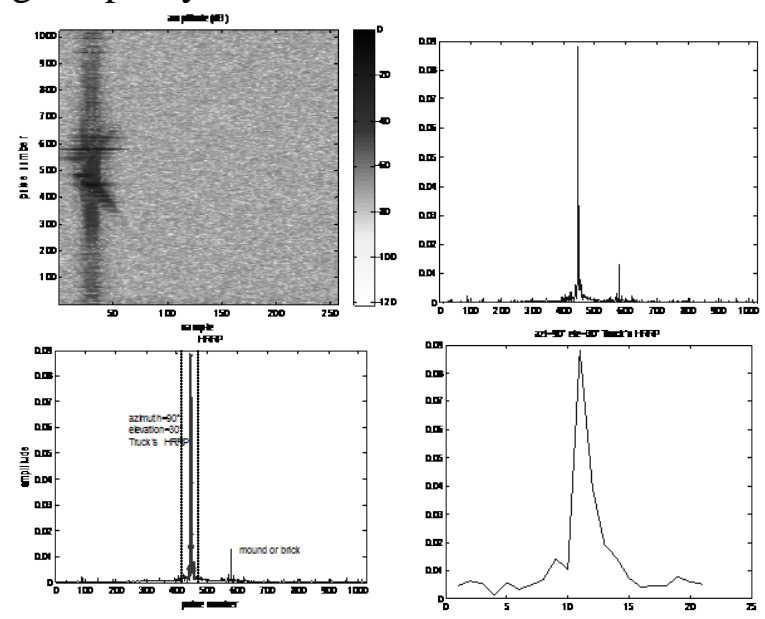

Fig.6. HRRP adaptive segmentation, (a)R-D image, (b)HRRP, (c)target's projection length is $2.9091 \mathrm{~m}\left(\mathrm{azi}=90^{\circ}\right.$, ele $\left.=30^{\circ}\right)$, HRRP peak position at $(477,0.088)$, rectangular range for intercepting is (437:457), (d)extractive HRRP in(c)

Truck's size is shown in Table 2. Refering to the Figure 4, we build matlab model to compute projection lenth $\mathrm{L}$. Then we get the intercept limit $\mathrm{N}=\mathrm{L} / 0.293$ around the HRRP peak under the current azimuth and elevation. The rectangular range for intercepting is the HRRP peak position $\pm \mathrm{N}$. As shown in Figure 6-d, extractive HRRP contains all the architectural feature of 6 merters range around the peak, avoiding the remote clutter interference. But, this method is invalid for the clutter overlapping with HRRP. It also doesn't work in two overlapping HRRPs. Further research needs to reference [7].

\subsection{HRRP Template Matching}

By means of the method in section 4.1, we build truck's and SUV's HRRP template library for experimental verification. In actual ground attack scene, we can get prior information by passive radar, then determine the target's rougn angle range, thus narrowing the scope of the template matching, so that we can reduce the computational complexity. Now we assume that the test target's elevation angle is knowm.

For an example in $30^{\circ}$ elevation, truck is the template library. SNR $=0 \mathrm{~dB}$, when we choose truck's HRRP in azimuth $0^{\circ}, 45^{\circ}, 90^{\circ}, 135^{\circ}, 180^{\circ}, 225^{\circ}, 270^{\circ}$ and $315^{\circ}$ to match truck, the result is shown in Figure 7, the successful matched value is almost close to 1 . If we chose SUV, the results is shown in Figure 8, the max is 0.84 .

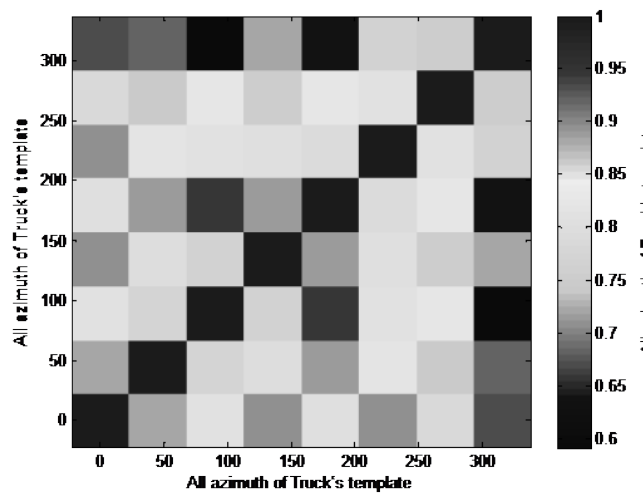

Fig.7. Truck match truck

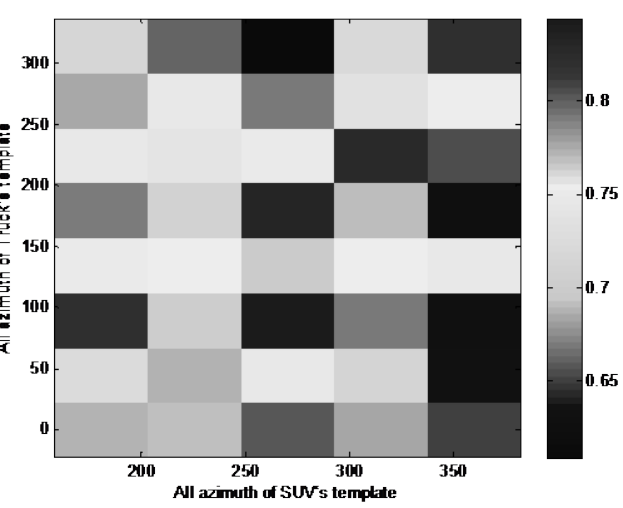

Fig.8. SUV match truck 
Truck is template library. SNR=10dB. We choose randomly one HRRP of truck/ SUV as test target in $10^{\circ} / 20^{\circ} / 30^{\circ}$ elevation, matching result is shown in Table 3. Truck's correct recognition rate is beyond $90 \%$, and SUV's false recognition rate is less than $1 \%$. The experiment result indicate that extractive HRRP by adaptive segmentation method is available in ground target recognition to judge target type and get target azimuth angle.

Table 3. Recognition rate for truck and SUV matching with truck

\begin{tabular}{l|c|c}
\hline \multirow{3}{*}{ Truck match truck } & Elevation $\left({ }^{\circ}\right)$ & Recognition rate \\
\cline { 2 - 3 } & 10 & $96.44 \%$ \\
\cline { 2 - 3 } & 20 & $98.69 \%$ \\
\hline \multirow{3}{*}{ SUV match truck } & 30 & $94.80 \%$ \\
\cline { 2 - 3 } & 10 & $0.12 \%$ \\
\cline { 2 - 3 } & 20 & $0.00 \%$ \\
\hline
\end{tabular}

\section{Conclusion}

This paper adopts ground measure data outfield, sets a rectangular window around the HRRP peak according to the projection length before matching, get rid of the clutter outside the window, and use the rest HRRP to match. Finally we verify the feasibility of ground target recognition based on HRRP, take the ground target recognition based on HRRP to the measured verification stage, lay the foundation for the algorithm improvement and engineering applications. In extractive HRRP, the sidelobe cover the weak scatters, we can extract the weak scatter around the strong ones by APC algorithm to improve the recognition rate. The thing to note is that the field experiment data is static, and the real data should be dynamic. Matching the dynamic data with static template library need the further research.

\section{Acknowledgments}

I would like to express my gratitude to all those who helped me during the writing of this thesis. My deepest gratitude goes foremost to Professor Song Zhiyong, my supervisor, for his constant encouragement and guidance. He has walked me through all the stages of the writing of this thesis. I also owe my sincere gratitude to my friends and my fellow classmates who gave me their help and time in listening to me and helping me work out my problems.

\section{References}

[1]Liu Jing. Study on Feature Extraction and Recognition Method for HRRP[D]. Xidian University, 2008: 8-35.

[2]Chen Feng, Du Lan, Liu Weihong. Radar HRRP Automatic Target Recognition Using Amplitude Information[J]. Acta Electronica Sinica, 2009, 3(3): 460-463.

[3]Yuan Zuxia, Gao Guiming. The Radar Target Recognition based on High Range Resolution Profiles[J]. Radar \& ECM, 2010, 3(30): 11-14.

[4]Xie Xin. Classification and Location about the Targets on the Ground for Missile-borne Radar[D]. Xidian University. Master's Thesis. 2012.

[5]Yin Kuiying. Study on SAR Image Pressing and Ground Target Recognition Technology[D]. Xidian University, 2011: 2-5.

[6]Jiang Yi. A Study on the Unmanned Aerial Vehicle Airborne SAR System Techniques[D]. Nanjing University of Science and Technology. Master's Thesis. 2004.

[7]Luo Xiaobo, Fan Hongqi, Song Zhiyong, Fu Qiang. Range Profiles Extraction for Maritime 
Form ation Targets based on Range-azimuth Clustering[J], 2013, 35(7): 1353-1361. 